OPEN ACCESS

Edited by:

Carlo Cattani,

Università degli Studi della Tuscia, Italy

Reviewed by:

Salih Diliali,

University of Chlef, Algeria

Sunil Kumar

National Institute of Technology

Jamshedpur, India

Aliyu Isa Aliyu,

Sun Yat-sen University, China

*Correspondence:

Behzad Ghanbar

b.ghanbary@yahoo.com

Specialty section:

This article was submitted to Mathematical Physics,

a section of the journal

Frontiers in Physics

Received: 10 October 2019 Accepted: 13 November 2019 Published: 06 December 2019

Citation:

Ghanbari B and Baleanu D (2019) New Solutions of Gardner's Equation

Using Two Analytical Methods.

Front. Phys. 7:202

doi: 10.3389/fphy.2019.00202

\section{New Solutions of Gardner's Equation Using Two Analytical Methods}

\author{
Behzad Ghanbari ${ }^{1,2 \star}$ and Dumitru Baleanu ${ }^{3,4,5}$ \\ ${ }^{1}$ Department of Engineering Science, Kermanshah University of Technology, Kermanshah, Iran, ${ }^{2}$ Department of \\ Mathematics, Faculty of Engineering and Natural Sciences, Bahçeşehir University, Istanbul, Turkey, ${ }^{3}$ Department of \\ Mathematics, Faculty of Arts and Sciences, Cankaya University, Ankara, Turkey, ${ }^{4}$ Department of Medical Research, China \\ Medical University Hospital, China Medical University, Taichung, Taiwan, ${ }^{5}$ Institute of Space Sciences, Bucharest, Romania
}

This article introduces and applies new methods to determine the exact solutions of partial differential equations that will increase our understanding of the capabilities of applied models in real-world problems. With these new solutions, we can achieve remarkable advances in science and technology. This is the basic idea in this article. To accurately describe this, some exact solutions to the Gardner's equation are obtained with the help of two new analytical methods including the generalized exponential rational function method and a Jacobi elliptical solution finder method. A set of new exact solutions containing four parameters is reported. The results obtained in this paper are new solutions to this equation that have not been introduced in previous literature. Another advantage of these methods is the determination of the varied solutions involving various classes of functions, such as exponential, trigonometric, and elliptic Jacobian. The three-dimensional diagrams of some of these solutions are plotted with specific values for their existing parameters. By examining these graphs, the behavior of the solution to this equation will be revealed. Mathematica software was used to perform the computations and simulations. The suggested techniques can be used in other real-world models in science and engineering.

Keywords: soliton solutions, generalized exponential rational function method, analytical solutions, PDE, computational, solitons, Gardner's equation

\section{INTRODUCTION}

It is difficult or impossible to determine the exact solution for many partial differential equations. In spite of these problems, in recent years a variety of efficient and practical methods have been proposed by mathematicians and physicists. Some of these methods are the exp-function method [1], the Darboux transformation [2], the Lie group analysis [3], the modified simple equation method [4], the homogeneous balance scheme [5], the sine-cosine method, and the tanh-coth method. Some new and effective attempts at determining solutions of partial differential equations can be found in [6-18].

The Gardner equation belongs to the category of integrable non-linear partial differential equations. The introduction of this equation is attributed to the famous mathematician Clifford Gardner in 1968 [19]. This equation can actually be generalized to the KdV equation. It is therefore sometimes referred to as the modified $\mathrm{KdV}$ equation. This equation is used in many areas of 
applications, such as hydrodynamics, plasma physics, and quantum field theory. This paper aims to employ two analytical methods to solve the following version of the integrable equation given by [20]

$$
u_{t}+k_{2} u u_{x}+k_{3} u^{2} u_{x}+k_{4} u_{x x x x}=0 .
$$

In this model, the dependent variable is $u(x, t)$, and The independent variables $x$ and $t$ are the spatial and temporal variables, respectively. Abdul-Majid Wazwaz in [21] has obtained some multiple-soliton solutions for a variant of the equation called the Gardner-KP (GKP) equation. His approach is based on the Hirota's bilinear method. In [22] the authors have applied the mapping method to study the dynamics of solitary waves governed by Gardner's equation. This equation arises while studying the shallow water waves. The perturbed Gardner equation is also discussed in this article through the aid of He's semi-inverse variational approach. Very recently, a classification of Lie symmetries for the Gardner equation has been reported in [23]. They have also used the similarity transformation method to introduce the invariant solutions. Their solutions are of multisoliton, compacton, negaton, positon, and kink wave soliton types. Considering some suitable auxiliary dependent variables, the authors of $[24,25]$ have obtained some exact invariant solutions for the equation with nonlocal symmetries. By using the method of planar dynamical systems approach, in different parameter regions, the authors in [26] have constructed the bifurcation of phase portraits of a traveling wave system. The work of [27] presents the ill-posedness results for the initial value problem for the Gardner equation. In [28], a certain classification of single traveling wave solutions of the time-fraction Gardner equation is investigated. These forms of the Gardner equation can be utilized to model various physical phenomena, such as the non-linear propagation of ion acoustic waves in an unmagnetized plasma.

As can be seen, numerous numerical and analytical methods have been used to study this equation. That proves the importance of this equation. This is our main motivation for writing this article - to determine new solutions to this equation This paper is organized as follows. The analysis of the GERFM is outlined in section 2. The application of the method of solving (1) is presented in section 3. Also, to have a better insight into the resulting solutions, many numerical simulations are carried out in this section. Finally, some remarks are discussed in the last section.

\section{THE ANALYSIS OF THE GERFM}

The GERFM has recently been applied to solve many nonlinear PDEs in some literature [29-31]. The successful use of this method in solving different sets of equations has made it an efficient method for solving partial equations. In order to gain insight into the method, let us have a quick review of the method. The steps to apply this method include the following.

1. Consider the following general non-linear PDE as

$$
\mathcal{N}\left(\psi, \psi_{x}, \psi_{t}, \psi_{x x}, \ldots\right)=0 .
$$

For two unknown constants of $\mu, v$, we define the new variables of $\psi=\psi(x)$ and $x=\mu x-v t$. then, Equation (28) can be reformulated as a non-linear ODE as

$$
\mathcal{N}\left(\psi, \mu \psi^{\prime},-v \psi^{\prime}, \mu^{2} \psi^{\prime \prime}, \ldots\right)=0 .
$$

2. Now, we take the solution Equation (29) into account for the following structure:

$$
\psi(\varkappa)=A_{0}+\sum_{k=1}^{M} A_{k} \Theta(\varkappa)^{k}+\sum_{k=1}^{M} B_{k} \Theta(\varkappa)^{-k} .
$$

where

$$
\Theta(\varkappa)=\frac{r_{1} e^{s_{1} \varkappa}+r_{2} e^{s_{2} \varkappa}}{r_{3} e^{s_{3} \varkappa}+r_{4} e^{s_{4} \varkappa}} .
$$

and $r_{i}, s_{i}(1 \leq i \leq 4), A_{0}, A_{k}$ and $B_{k}(1 \leq k \leq M)$ are unknown constants. Then, equating the two values of the amplitude, from (12) and (13), leads to the value of $M$.

3. Putting Equation (30) into Equation (29) and collecting all terms, the left-hand side of Equation (29) give us an algebraic equation $P\left(Z_{1}, Z_{2}, Z_{3}, Z_{4}\right)=0$ in terms of $Z_{i}=e^{s_{i} \chi}$ for $i=1, \ldots, 4$. Zeroing each coefficient of $P$, we get a system of non-linear equations in terms of $r_{i}, s_{i}(1 \leq i \leq 4)$, and $\mu, l, A_{0}, A_{k}$ and $B_{k}(1 \leq k \leq M)$.

4. Any symbolic computation software can be utilized to solve this system to determine the values of $r_{i}, s_{i}(1 \leq i \leq 4), A_{0}, A_{k}$, and $B_{k}(1 \leq k \leq M)$. Using these results will direct us to soliton solutions of the main non-linear PDE.

\section{APPLICATION OF THE METHOD}

Below, we present a detailed presentation of the solution of Equation (1). To this end, let us consider the following new definitions

$$
u(x, t)=\mathcal{U}(\varkappa), \quad \varkappa=\mu x-v t,
$$

where $\mu$ and $v$ are arbitrary unknown parameters. Utilizing the wave transformation (36) converts Equation (1) into the following single NODE:

$$
\left(k_{1} v-\mu\right) \mathcal{U}^{\prime}+k_{2} v \mathcal{U} \mathcal{U}^{\prime}+k_{3} v \mathcal{U}^{2} \mathcal{U}^{\prime}+k_{4} v^{3} \mathcal{U}^{\prime \prime \prime}=0,
$$

Performing the integral with respect to $\varkappa$ and with $c=0$, the last equation becomes

$$
\left(k_{1} v-\mu\right) \mathcal{U}+\frac{1}{3} k_{2} v \mathcal{U}^{2}+\frac{1}{3} k_{3} v \mathcal{U}^{3}+k_{4} v^{3} \mathcal{U}^{\prime \prime}=0 .
$$


Then, equating the two values of $3 M$ and $M+2$, corresponding to $\mathcal{U}^{3}$ and $\mathcal{U}^{\prime \prime}$ in Equation (8), leads to the value of $M=1$. Using Equation (5) together with $M=1$, we have

$$
\mathcal{U}(\varkappa)=A_{0}+A_{1} \Theta(\varkappa)+\frac{B_{1}}{\Theta(\varkappa)} .
$$

Proceeding as outlined in the second section and depending on the values of the parameters we obtain in the solitary wave solutions.

\section{Set 1:}

One obtains $r=[-3,-1,1,1]$ along with $s=[2,0,2,0]$, so (5) turns to

$$
\Theta(\varkappa)=\frac{-3 \mathrm{e}^{2 \varkappa}-1}{\mathrm{e}^{2 \varkappa}+1} .
$$

In this case we obtain two exact solutions, as:

I.

$$
\begin{aligned}
& \mu=\frac{-k_{2}^{2} k_{2} \sqrt{-6 k_{3} k_{4}}}{72 k_{3}^{2} k_{4}}, v=\frac{k_{2} \sqrt{-6 k_{3} k_{4}}}{12 k_{3} k_{4}}, \\
& A_{0}=\frac{k_{2}}{2 k_{3}}, A_{1}=0, B_{1}=\frac{3 k_{2}}{2 k_{3}} .
\end{aligned}
$$

Putting these values in Equations (10) and (37) yields a solitary wave solution for Equation (1) as:

$$
u_{1}(x, t)=-\frac{k_{2}}{k_{3}\left(3 \mathrm{e}^{2 \varkappa}+1\right)},
$$

where

$$
\varkappa=\frac{-k_{3}^{2} \sqrt{-6 k_{3} k_{4}}}{72 k_{3}^{2} k_{4}} x-\frac{k_{2} \sqrt{-6 k_{3} k_{4}}}{6 k_{3} k_{4}} t
$$

II.

$$
\begin{aligned}
\mu & =\frac{-k_{2}^{2} k_{2} \sqrt{-6 k_{3} k_{4}}}{72 k_{3}^{2} k_{4}}, v=\frac{k_{2} \sqrt{-6 k_{3} k_{4}}}{12 k_{3} k_{4}}, \\
A_{0} & =-\frac{3 k_{2}}{2 k_{3}}, A_{1}=0, B_{1}=-\frac{3 k_{2}}{2 k_{3}} .
\end{aligned}
$$

Putting these values in Equations (10) and (37) yields a solitary wave solution for Equation (1) as:

$$
u_{2}(x, t)=-\frac{3 k_{2} \mathrm{e}^{2 \varkappa}}{k_{3}\left(3 \mathrm{e}^{2 \varkappa}+1\right)},
$$

where

$$
\varkappa=\frac{-k_{3}^{2} \sqrt{-6 k_{3} k_{4}}}{36 k_{3}^{2} k_{4}} x-\frac{k_{2} \sqrt{-6 k_{3} k_{4}}}{6 k_{3} k_{4}} t
$$

Set 2:

One obtains $r=[-1,3,1,-1]$ along with $s=[1,-1,1,-1]$, so (5) turns to

$$
\Theta(\varkappa)=\frac{\cosh (\varkappa)-2 \sinh (\varkappa)}{\sinh (\varkappa)} .
$$

In this case we obtain two exact solutions, as:

I.

$$
\begin{aligned}
\mu=\frac{-k_{2}^{2} k_{2} \sqrt{-6 k_{3} k_{4}}}{576 k_{3}^{2} k_{4}}, v & =\frac{k_{2} \sqrt{-6 k_{3} k_{4}}}{24 k_{3} k_{4}}, A_{0}=-\frac{k_{2}}{k_{3}}, \\
A_{1} & =-\frac{k_{2}}{4 k_{3}}, B_{1}=-\frac{3 k_{2}}{4 k_{3}} .
\end{aligned}
$$

Now, from Equations (13) and (37) we will reach to a solitary wave solution for Equation (1) as:

$$
u_{3}(x, t)=-\frac{k_{2}}{2 k_{3}\left(\sinh (2 \varkappa)-4 \sinh ^{2}(\varkappa)\right)},
$$

where

$$
\varkappa=\frac{-k_{3}^{2} \sqrt{-6 k_{3} k_{4}}}{576 k_{3}^{2} k_{4}} x-\frac{k_{2} \sqrt{-6 k_{3} k_{4}}}{24 k_{3} k_{4}} t
$$

II.

$$
\begin{aligned}
\mu & =\frac{k_{2} \sqrt{-3 k_{3} k_{4}(\sqrt{7}+4)}\left(406 k_{2}^{2}-56 k_{2}^{2} \sqrt{7}\right)}{1944 k_{3}{ }^{2} k_{4}(5 \sqrt{7}-7)(\sqrt{7}+1)^{2}}, \\
v & =\frac{k_{2} \sqrt{-3 k_{3} k_{4}(\sqrt{7}+4)}}{36 k_{3} k_{4}}, \\
A_{0} & =-\frac{k_{2}(5 \sqrt{7}+11)}{6 k_{3}(\sqrt{7}+1)}, A_{1}=-\frac{k_{2}(\sqrt{7}+1)}{12 k_{3}}, \\
B_{1} & =\frac{-59 k_{2} \sqrt{7}-119 k_{2}}{k_{3}(\sqrt{7}+1)^{3}(5 \sqrt{7}-7)} .
\end{aligned}
$$

Equations (13) and (37) for these values will introduce a solitary wave solution for Equation (1) as:

$$
\begin{aligned}
& u_{4}(x, t) \\
& =-\frac{2 k_{2}}{3 k_{3}} \frac{(116 \sqrt{7}-112) \cosh ^{2}(\varkappa)-(29 \sqrt{7}-28) \sinh (2 \varkappa)}{(5 \sqrt{7}-7)(\sqrt{7}+1)^{3}\left(\sinh (2 \varkappa)-4 \sinh ^{2}(\varkappa)\right)},
\end{aligned}
$$

where

$$
\begin{gathered}
\varkappa=\frac{k_{2} \sqrt{-3 k_{3} k_{4}(\sqrt{7}+4)}\left(406 k_{2}^{2}+1404 \sqrt{7} k_{1} k_{3}\right.}{\left.-56 k_{2}^{2} \sqrt{7}+756 k_{1} k_{3}\right)} \\
-\frac{1944 k_{3}{ }^{2} k_{4}(5 \sqrt{7}-7)(\sqrt{7}+1)^{2}}{36 k_{3} k_{4}} t .
\end{gathered}
$$

Set 3:

One obtains $r=[3,2,1,1]$ along with $s=[1,0,1,0]$, so (5) turns to

$$
\Theta(\varkappa)=\frac{3 \mathrm{e}^{\varkappa}+2}{\mathrm{e}^{\varkappa}+1} .
$$


In this case we obtain an exact solution, as:

I.

$$
\begin{aligned}
\mu & =\frac{-k_{2}^{3} \sqrt{-6 k_{3} k_{4}}}{4500 k_{3}^{2} k_{4}}, v=\frac{k_{2} \sqrt{-6 k_{3} k_{4}}}{30 k_{3} k_{4}}, \\
A_{0} & =-\frac{k_{2}}{k_{3}}, A_{1}=\frac{k_{2}}{5 k_{3}}, B_{1}=\frac{6 k_{2}}{5 k_{3}} .
\end{aligned}
$$

Putting these values in Equations (16) and (37) yields a solitary wave solution for Equation (1) as:

$$
u_{5}(x, t)=-\frac{k_{2} \mathrm{e}^{\varkappa}}{5 k_{3}\left(1+\mathrm{e}^{\varkappa}\right)\left(3 \mathrm{e}^{\varkappa}+2\right)},
$$

where

$$
\varkappa=\frac{\left(150 k_{1} k_{3}-k_{2}^{2}\right) k_{2} \sqrt{-6 k_{3} k_{4}}}{4500 k_{3}^{2} k_{4}} x-\frac{k_{2} \sqrt{-6 k_{3} k_{4}}}{30 k_{3} k_{4}} t
$$

Set 5:

One obtains $r=[1,1,1,-1]$ along with $s=[2,0,2,0]$, so (5) turns to

$$
\Theta(\varkappa)=\frac{\mathrm{e}^{2 \varkappa}+1}{\mathrm{e}^{2 \varkappa}-1} .
$$

In this case we obtain an exact solution, as:

I.

$$
\begin{aligned}
\mu & =\frac{-k_{3}^{2} \sqrt{-6 k_{3} k_{4}}}{144 k_{3}^{2} k_{4}}, v=\frac{k_{2} \sqrt{-6 k_{3} k_{4}}}{24 k_{3} k_{4}}, \\
A_{0} & =-\frac{k_{2}}{2 k_{3}}, A_{1}=-\frac{k_{2}}{4 k_{3}}, B_{1}=-\frac{k_{2}}{4 k_{3}} .
\end{aligned}
$$

For these solutions in Equations (18) and (37) yields a solitary wave solution for Equation (1) as:

$$
u_{6}(x, t)=-\frac{k_{2} \mathrm{e}^{4 \varkappa}}{k_{3}\left(\mathrm{e}^{4 \varkappa}-1\right)},
$$

where

$$
\varkappa=\frac{-k_{3}^{2} \sqrt{-6 k_{3} k_{4}}}{36 k_{3}^{2} k_{4}} x-\frac{k_{2} \sqrt{-6 k_{3} k_{4}}}{6 k_{3} k_{4}} t
$$

\section{Set 6:}

One obtains $r=[-2-i, 2-i,-1,1]$ along with $s=[i,-i, i,-i]$, so (5) turns to

$$
\Theta(\varkappa)=\frac{\cos (x)+2 \sin (x)}{\sin (x)} .
$$

In this case we obtain an exact solution, as:
I.

$$
\begin{aligned}
\mu & =\frac{k_{3}^{2} \sqrt{-6 k_{3} k_{4}}}{576 k_{3}^{2} k_{4}}, v=\frac{k_{2} \sqrt{-6 k_{3} k_{4}}}{24 k_{3} k_{4}}, \\
A_{0} & =-\frac{k_{2}}{k_{3}}, A_{1}=\frac{k_{2}}{4 k_{3}}, B_{1}=\frac{5 k_{2}}{4 k_{3}} .
\end{aligned}
$$

Inserting these values in Equations (20) and (37) yields a solitary wave solution for Equation (1) as:

$$
u_{7}(x, t)=\frac{k_{2}}{2 k_{3}\left(\sin (2 \varkappa)+4 \sin ^{2}(\varkappa)\right)},
$$

where

$$
\varkappa=\frac{k_{2}^{3} \sqrt{-6 k_{3} k_{4}}}{576 k_{3}^{2} k_{4}} x-\frac{k_{2} \sqrt{-6 k_{3} k_{4}}}{24 k_{3} k_{4}} t .
$$

Set 7:

One obtains $r=[-3,-1,1,1]$ along with $s=[1,-1,1,-1]$, so (5) turns to

$$
\Theta(\varkappa)=\frac{-2 \cosh (\varkappa)-\sinh (x)}{\cosh (\varkappa)} .
$$

In this case we obtain an exact solution, as:

I.

$$
\begin{aligned}
\mu & =\frac{-k_{3}^{2} \sqrt{-6 k_{3} k_{4}}}{72 k_{3}^{2} k_{4}}, v=\frac{k_{2} \sqrt{-6 k_{3} k_{4}}}{12 k_{3} k_{4}}, \\
A_{0} & =-\frac{3 k_{2}}{3 k_{3}}, A_{1}=0, B_{1}=-\frac{3 k_{2}}{2 k_{3}} .
\end{aligned}
$$

Putting these values in Equations (22) and (37) yields a solitary wave solution for Equation (1) as:

$$
u_{8}(x, t)=-\frac{3 k_{2}(\cosh (\varkappa)+\sinh (\varkappa))}{2 k_{3}(2 \cosh (\varkappa)+\sinh (\varkappa))},
$$

where

$$
\varkappa=\frac{-k_{3}^{2} \sqrt{-6 k_{3} k_{4}}}{72 k_{3}^{2} k_{4}} x-\frac{k_{2} \sqrt{-6 k_{3} k_{4}}}{12 k_{3} k_{4}} t .
$$

Set 8:

One obtains $r=[1+i, 1-i, 1,1]$ along with $s=[i,-i, i,-i]$, so (5) turns to

$$
\Theta(\varkappa)=\frac{-\sin (\varkappa)+\cos (x)}{\cos (x)} .
$$

In this case we obtain an exact solution, as:

I.

$$
\begin{aligned}
& \mu=\frac{k_{2}\left(-374 k_{2}^{2}-6 k_{2}^{2} \sqrt{17}\right) \sqrt{-3 k_{3} k_{4}(\sqrt{17}+9)}}{9216 k_{3}^{2}(\sqrt{17}+1)^{2} k_{4}}, \\
& v=\frac{k_{2} \sqrt{-3 k_{3} k_{4}(\sqrt{17}+9)}}{48 k_{3} k_{4}}
\end{aligned}
$$




$$
\begin{aligned}
& A_{0}=-\frac{k_{2}(5 \sqrt{17}+13)}{8 k_{3}(\sqrt{17}+1)}, A_{1}=\frac{k_{2}(\sqrt{17}+1)}{16 k_{3}}, \\
& B_{1}=\frac{k_{2}(\sqrt{17}+1)}{8 k_{3}} .
\end{aligned}
$$

Using the above solutions in Equations (24) and (37) yields a solitary wave solution for Equation (1) as:

$$
u_{9}(x, t)=-\frac{k_{2}}{8 k_{3}} \frac{(6 \sqrt{17}-10) \cos ^{3}(\varkappa)-4(\sqrt{17}+1) \cos (\varkappa)}{(\sqrt{17}+1)\left(2 \cos (\varkappa)^{3}-\cos (\varkappa)\right)},
$$

where

$$
\begin{gathered}
\varkappa=\frac{k_{2}\left(-374 k_{2}^{2}-6 k_{2}^{2} \sqrt{17}\right) \sqrt{-3 k_{3} k_{4}(\sqrt{17}+9)}}{9216 k_{3}^{2}(\sqrt{17}+1)^{2} k_{4}} x \\
-\frac{k_{2} \sqrt{-3 k_{3} k_{4}(\sqrt{17}+9)}}{48 k_{3} k_{4}} t .
\end{gathered}
$$

Set 9:

One obtains $r=[-1,-2,1,1]$ along with $s=[1,0,1,0]$, so (5) turns to

$$
\Theta(\varkappa)=\frac{-\mathrm{e}^{\varkappa}-2}{\mathrm{e}^{\varkappa}+1} .
$$

In this case we obtain an exact solution, as:

I.

$$
\begin{aligned}
\mu & =\frac{k_{2}\left(771 k_{2}^{2} \sqrt{73}-2263 k_{2}^{2}\right) \sqrt{-3 k_{3} k_{4}(3 \sqrt{73}+41)}}{147456 k_{3}^{2}(\sqrt{73}+3)^{2} k_{4}} \\
v & =\frac{k_{2} \sqrt{-3 k_{3} k_{4}(3 \sqrt{73}+41)}}{96 k_{3} k_{4}} \\
A_{0} & =-\frac{k_{2}(25 \sqrt{73}+171)}{32 k_{3}(\sqrt{73}+3)}, A_{1}=-\frac{k_{2}(\sqrt{73}+3)}{32 k_{3}} \\
B_{1} & =-\frac{k_{2}(\sqrt{73}+3)}{16 k_{3}}
\end{aligned}
$$

Inserting these values in Equations (26) and (37) yields a solitary wave solution for Equation (1) as:

$$
\begin{aligned}
& u_{10}(x, t) \\
& =-\frac{k_{2}\left((7 \sqrt{73}-75) \mathrm{e}^{2 \varkappa}+(27 \sqrt{73}-143) \mathrm{e}^{\varkappa}+14 \sqrt{73}-150\right)}{32 k_{3}(\sqrt{73}+3)\left(1+\mathrm{e}^{\varkappa}\right)\left(\mathrm{e}^{\varkappa}+2\right)},
\end{aligned}
$$

where

$$
=\frac{k_{2}\left(\left(9216 \sqrt{73}+(771 \sqrt{73}-2263) k_{2}^{2}\right) \sqrt{-3 k_{3} k_{4}(3 \sqrt{73}+41)}\right.}{147456 k_{3}^{2}(\sqrt{73}+3)^{2} k_{4}} x
$$

$$
-\frac{k_{2} \sqrt{-3 k_{3} k_{4}(3 \sqrt{73}+41)}}{96 k_{3} k_{4}} t .
$$

It is worth mentioning that the necessary condition to establish the existence of the acquired solutions $u_{1}(x, t)-u_{10}(x, t)$ is $k_{3} k_{4}<0$.

\section{A JACOBI ELLIPTICAL SOLUTIONS FINDER METHOD}

In this part, we are going to obtain new exact soliton solutions to the equation under investigation, using a newly proposed method [32]. To this end, we will briefly review the steps of using the method.

1. The main purpose of this method is to solve an equation as follows:

$$
\mathcal{N}\left(\phi, \phi_{x}, \phi_{t}, \phi_{x x}, \ldots\right)=0 .
$$

2. Defining $\phi=\phi(x)$ and $x=\mu x-l t$, Equation (28) is converted to

$$
\mathcal{N}\left(\phi, \phi^{\prime}, \phi^{\prime \prime}, \ldots\right)=0,
$$

where $\mu$ and $l$ are two constants.

3. At this point, the symbolic form of the Equation (29) can be formulated as follows:

$$
\phi(\varkappa)=\frac{\alpha_{0}+\sum_{k=1}^{2 N} \alpha_{k} \Theta(\varkappa)^{k}}{\beta_{0}+\sum_{k=1}^{2 N} \beta_{k} \Theta(\varkappa)^{k}},
$$

where the values of constants $A_{0}, B_{0}$ and $A_{k}, B_{k}(1 \leq k \leq 2 N)$ are so that (30) is a solution to the Equation (29).

4. The value of $N$ in Equation (30) is obtained using the balance principles and $\Theta(\varkappa)$ satisfies the following non-linear ODE:

$$
\begin{aligned}
& \Theta(\varkappa)^{\prime 2}=h_{0}+h_{2} \Theta(\varkappa)^{2}+h_{4} \Theta(\varkappa)^{4}+h_{6} \Theta(\varkappa)^{6}, \\
& \Theta(\varkappa)^{\prime \prime}=h_{2} \Theta(\varkappa)+2 h_{4} \Theta(\varkappa)^{3}+3 h_{6} \Theta(\varkappa)^{5},
\end{aligned}
$$

where $h_{i}(i=0,2,4,6)$ are real constants.

5. The solution of the Equation (31) should be as follows

$$
\Theta(\varkappa)=\frac{\Phi(\varkappa)}{\sqrt{f \Phi(\varkappa)^{2}+g}},
$$

where $\Phi(\varkappa)^{2}+g>0$, and $\Phi(\varkappa)$ is the solution of the Jacobian elliptic equation

$$
\Phi(\varkappa)^{\prime 2}=l_{0}+l_{2} \Phi(\varkappa)^{2}+l_{4} \Phi(\varkappa)^{4},
$$

and $l_{j}(j=0,2,4)$ are constants need to be calculated, The relationships for $f$ and $g$ will also be as follows:

$$
\begin{aligned}
& f=\frac{h_{4}\left(l_{2}-h_{2}\right)}{\left(l_{2}-h_{2}\right)^{2}+3 l_{0} l_{4}-2 l_{2}\left(l_{2}-h_{2}\right)}, \\
& g=\frac{3 h_{4} l_{0}}{\left(l_{2}-h_{2}\right)^{2}+3 l_{0} l_{4}-2 l_{2}\left(l_{2}-h_{2}\right)},
\end{aligned}
$$


under the constraint condition

$$
h_{2}^{4}\left(l_{2}-h_{2}\right)\left[9 l_{0} l_{4}-\left(l_{2}-h_{2}\right)\left(2 l_{2}+h_{2}\right)\right]+3 h_{6}\left[3 l_{0} l_{4}-\left(l_{2}^{2}-h_{2}^{2}\right)\right]^{2}=0 .
$$

6. It is known that solutions of Equation (33) are in terms of Jacobi elliptic solutions. Inserting both (33) and (32) into Equation (30), one gets the optical solutions of Equation (28). It should be noted that by using the limits in Table 2, the Jacobian elliptic functions used in the solutions reduce to the known triangular functions.

\section{THE APPLICATION OF THE METHOD}

In this section, to begin solving the equation, we first introduce the following new variables

$$
\phi=\mathcal{U}(\varkappa), \quad \varkappa=\mu x-v t .
$$

Then we will consider the balancing principles in Equation (8). So, one gets $N=1$. So, the Equation (30) can be rewritten as follows

$$
\mathcal{U}(\varkappa)=\frac{\alpha_{0}+\alpha_{1} \Theta(\varkappa)+\alpha_{2} \Theta^{2}(\varkappa)}{\beta_{0}+\beta_{1} \Theta(\varkappa)+\beta_{2} \Theta^{2}(\varkappa)} .
$$

The following results will be obtained using the method presented in section 4 of this article.

Set 11: We attain

$$
\begin{gathered}
\mu=\frac{-2 v k_{2}^{2}}{27 k_{3}}, v=v, \alpha_{0}=-\frac{\beta_{0} k_{2}}{3 k_{3}}, \alpha_{1}=\alpha_{1}, \alpha_{2}=0, \\
\beta_{0}=\beta_{0}, \beta_{1}=0, \beta_{2}=0, \\
h_{0}=h_{0}, h_{2}=\frac{k_{2}^{2}}{27 v^{2} k_{3} k_{4}}, h_{4}=-\frac{\alpha_{1}{ }^{2} k_{3}}{6 v^{2} \beta_{0}{ }^{2} k_{4}}, h_{6}=0 .
\end{gathered}
$$

\begin{tabular}{|c|c|c|}
\hline Function & $m \rightarrow 0$ & $m \rightarrow 1$ \\
\hline $\operatorname{sn}(\varkappa)=\operatorname{sn}(\varkappa, m)$ & $\sin (x)$ & $\tanh (x)$ \\
\hline$c n(\varkappa)=c n(\varkappa, m)$ & $\cos (x)$ & $\operatorname{sech}(x)$ \\
\hline$d n(\varkappa)=d n(\varkappa, m)$ & 1 & $\operatorname{sech}(x)$ \\
\hline$n s(\varkappa)=n s(\varkappa, m)$ & $\csc (\varkappa)$ & $\operatorname{coth}(x)$ \\
\hline $\operatorname{cs}(\varkappa)=\operatorname{cs}(\varkappa, m)$ & $\cot (\varkappa)$ & $\operatorname{csch}(x)$ \\
\hline$d s(\varkappa)=d s(\varkappa, m)$ & $\csc (\varkappa)$ & $\operatorname{csch}(x)$ \\
\hline$s c(\varkappa)=s c(\varkappa, m)$ & $\tan (\varkappa)$ & $\sinh (x)$ \\
\hline$s d(\varkappa)=s d(\varkappa, m)$ & $\sin (x)$ & $\sinh (x)$ \\
\hline$n c(\varkappa)=n c(\varkappa, m)$ & $\sec (x)$ & $\cosh (x)$ \\
\hline$c d(\varkappa)=c d(\varkappa, m)$ & $\cos (\varkappa)$ & 1 \\
\hline$n d(x)=n d(x, m)$ & 1 & $\cosh (x)$ \\
\hline
\end{tabular}

Using No. 1 in Table 1 we have
TABLE 1 | Jacobi elliptic solutions of Equation (33).

\begin{tabular}{lcccc}
\hline No & $\mathbf{I}_{\mathbf{0}}$ & $\mathbf{I}_{\mathbf{2}}$ & $\mathbf{I}_{\mathbf{4}}$ & $\boldsymbol{\Theta}(\varkappa)$ \\
\hline 1 & 1 & $-\left(1+m^{2}\right)$ & $m^{2}$ & $s n(\varkappa, m) \operatorname{or} c d(\varkappa, m)$ \\
2 & $1-m^{2}$ & $2 m^{2}-1$ & $-m^{2}$ & $\operatorname{cn}(\varkappa, m)$ \\
3 & $m^{2}-1$ & $2-m^{2}$ & -1 & $d n(\varkappa, m)$ \\
4 & $m^{2}$ & $-\left(m^{2}+1\right)$ & 1 & $n s(\varkappa, m)$ or $d c(\varkappa, m)$ \\
5 & $-m^{2}$ & $2 m^{2}-1$ & $1-m^{2}$ & $n c(\varkappa, m)$ \\
6 & -1 & $2-m^{2}$ & $-\left(1-m^{2}\right)$ & $n d(\varkappa, m)$ \\
7 & 1 & $2-m^{2}$ & $1-m^{2}$ & $s c(\varkappa, m)$ \\
8 & 1 & $2 m^{2}-1$ & $-m^{2}\left(1-m^{2}\right)$ & $s d(\varkappa, m)$ \\
9 & $1-m^{2}$ & $2-m^{2}$ & 1 & $c s(\varkappa, m)$ \\
10 & $-m^{2}\left(1-m^{2}\right)$ & $2 m^{2}-1$ & 1 & $d s(\varkappa, m)$ \\
11 & $\frac{1-m^{2}}{4}$ & $\frac{1+m^{2}}{2}$ & $\frac{1-m^{2}}{4}$ & $n c(\varkappa, m) \pm s c(\varkappa, m)$ or \\
& $\frac{-\left(1-m^{2}\right)^{2}}{4}$ & $\frac{m^{2}+1}{2}$ & $-\frac{1}{4}$ & $\frac{c m(x, m)}{1 \pm \pm n(x, m)}$ \\
12 & $\frac{1}{4}$ & $\frac{1-2 m^{2}}{2}$ & $\frac{1}{4}$ & $\frac{s n(\varkappa, m) \pm d n(\varkappa, m)}{1 \pm n,(x, m)}$ \\
13 & $\frac{1}{4}$ & $\frac{1+m^{2}}{2}$ & $\frac{\left(1-m^{2}\right)^{2}}{4}$ & $\frac{s n(x, m)}{\operatorname{cn}(x, m) \pm d n(x, m)}$ \\
14 & $\frac{1}{4}$ & &
\end{tabular}

TABLE 2 | Jacobi elliptic functions and their limits.

provided that

$$
\mathcal{U}(\varkappa)=-\frac{k_{2}}{3 k_{3}}+\alpha_{1} \sqrt{6} \sqrt{-\frac{(s n(\varkappa, m))^{2} v^{2} k_{4}}{\alpha_{1}{ }^{2} k_{3}} \frac{\left(m^{4}-m^{2}-\frac{k_{2}{ }^{4}}{729 v^{4} k_{3}{ }^{2} k_{4}{ }^{2}}+1\right)}{-3+\left(m^{2}+\frac{k_{2}{ }^{2}}{27 v^{2} k_{3} k_{4}}+1\right)(\operatorname{sn}(\varkappa, m))^{2}}},
$$

$$
\begin{aligned}
& \left(27 m^{2} v^{2} k_{3} k_{4}+27 v^{2} k_{3} k_{4}+k_{2}^{2}\right) \\
& \left(27 m^{2} v^{2} k_{3} k_{4}-54 v^{2} k_{3} k_{4}+k_{2}^{2}\right) \\
& \left(54 m^{2} v^{2} k_{3} k_{4}-27 v^{2} k_{3} k_{4}-k_{2}^{2}\right)=0 .
\end{aligned}
$$

The exact soliton solution to the equation will thus be determined as follows

where

$$
u_{11}(x, t)=-\frac{k_{2}}{3 k_{3}}+\alpha_{1} \sqrt{6} \sqrt{-\frac{(s n(\varkappa, m))^{2} v^{2} k_{4}}{\alpha_{1}{ }^{2} k_{3}} \frac{\left(m^{4}-m^{2}-\frac{k_{2}{ }^{4}}{729 v^{4} k_{3}{ }^{2} k_{4}{ }^{2}}+1\right)}{-3+\left(m^{2}+\frac{k_{2}^{2}}{27 v^{2} k_{3} k_{4}}+1\right)(\operatorname{sn}(\varkappa, m))^{2}}},
$$

$$
\varkappa=\frac{-2 v k_{2}^{2}}{27 k_{3}} x-v t
$$


Using No. 2 in Table 1 we have

$$
\mathcal{U}(\varkappa)=-\frac{k_{2}}{3 k_{3}}+\alpha_{1} \sqrt{6} \sqrt{-\frac{k_{4} v^{2}\left((\operatorname{sn}(\varkappa, m))^{2}-1\right)\left(m^{4}-m^{2}-\frac{k_{2}{ }^{4}}{729 v^{4} k_{3}{ }^{2} k_{4}{ }^{2}}+1\right)}{\alpha_{1}{ }^{2} k_{3}\left(2(c n(\varkappa, m))^{2} m^{2}-\frac{(c n(\varkappa, m))^{2} k_{2}{ }^{2}}{27 v^{2} k_{3} k_{4}}-(c n(\varkappa, m))^{2}-3 m^{2}+3\right)}},
$$

provided that

$$
\left(27 m^{2} v^{2} k_{3} k_{4}+27 v^{2} k_{3} k_{4}+k_{2}^{2}\right)\left(27 m^{2} v^{2} k_{3} k_{4}-54 v^{2} k_{3} k_{4}+k_{2}^{2}\right)\left(54 m^{2} v^{2} k_{3} k_{4}-27 v^{2} k_{3} k_{4}-k_{2}^{2}\right)=0
$$

The exact soliton solution to the equation will thus be determined as follows

$$
u_{12}(x, t)=-\frac{k_{2}}{3 k_{3}}+\alpha_{1} \sqrt{6} \sqrt{-\frac{k_{4} v^{2}\left((\operatorname{sn}(\varkappa, m))^{2}-1\right)\left(m^{4}-m^{2}-\frac{k_{2}^{4}}{729 v^{4} k_{3}^{2} k_{4}^{2}}+1\right)}{\alpha_{1}^{2} k_{3}\left(2(c n(\varkappa, m))^{2} m^{2}-\frac{(c n(\varkappa, m))^{2} k_{2}^{2}}{27 v^{2} k_{3} k_{4}}-(c n(\varkappa, m))^{2}-3 m^{2}+3\right)}},
$$

where

$$
x=\frac{-2 v k_{2}^{2}}{27 k_{3}} x-v t .
$$

Set 12: We attain

$$
\begin{gathered}
\mu=\frac{-2 v k_{2}^{2}}{27 k_{3}}, v=v, \alpha_{0}=\alpha_{0}, \alpha_{1}=\alpha_{1}, \alpha_{2}=0, \beta_{0}=0, \beta_{1}=-\frac{3 k_{3} \alpha_{1}}{k_{2}}, \beta_{2}=0, \\
h_{0}=-\frac{\alpha_{0}{ }^{2} k_{2}^{2}}{54 \alpha_{1}{ }^{2} k_{3} v^{2} k_{4}}, h_{2}=\frac{k_{2}^{2}}{27 v^{2} k_{3} k_{4}}, h_{4}=h_{4}, h_{6}=0 .
\end{gathered}
$$

Using No. 1 in Table 1 we have

$$
\mathcal{U}(\varkappa)=-\frac{k_{2}}{3 k_{3} \alpha_{1}} \frac{\left(\alpha_{1}{ }^{\left.\frac{(s n(\varkappa, m))^{2}}{h_{4}} \frac{\left(m^{4}-m^{2}-\frac{k_{2}{ }^{4}}{729 v^{4} k_{3}{ }^{2} k_{4}^{2}}+1\right)}{\left(-3+\left(m^{2}+\frac{k_{2}^{2}}{27 v^{2} k_{3} k_{4}}+1\right)(\operatorname{sn}(\varkappa, m))^{2}\right)}+\alpha_{0}\right)}\right.}{\sqrt{\frac{(s n(\varkappa, m))^{2}}{h_{4}}\left(m^{4}-m^{2}-\frac{k_{2}{ }^{4}}{729 v^{4} k_{3}{ }^{2} k_{4}^{2}}+1\right)\left(-3+\left(m^{2}+\frac{k_{2}^{2}}{27 v^{2} k_{3} k_{4}}+1\right)(\operatorname{sn}(\varkappa, m))^{2}\right)}}
$$

provided that

$$
\left(27 m^{2} v^{2} k_{3} k_{4}-54 v^{2} k_{3} k_{4}+k_{2}^{2}\right)\left(54 m^{2} v^{2} k_{3} k_{4}-27 v^{2} k_{3} k_{4}-k_{2}^{2}\right)\left(27 m^{2} v^{2} k_{3} k_{4}+27 v^{2} k_{3} k_{4}+k_{2}^{2}\right)=0
$$

The exact soliton solution to the equation will thus be determined as follows

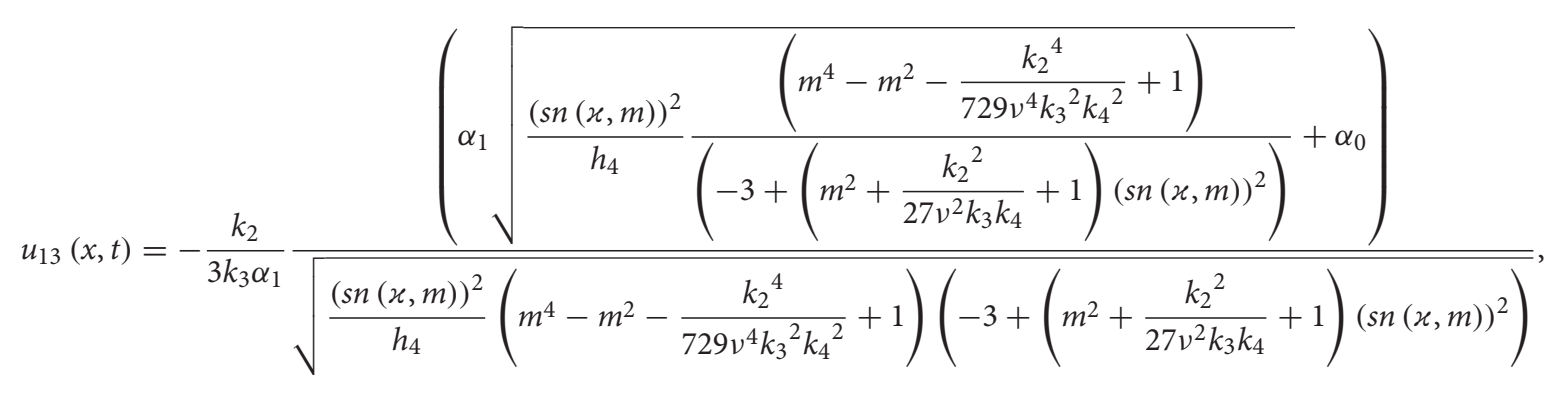


where

$$
\varkappa=\frac{-2 v k_{2}^{2}}{27 k_{3}} x-v t
$$

Using No. 4 in Table 1 we have



provided that

$$
\left(27 m^{2} v^{2} k_{3} k_{4}-54 v^{2} k_{3} k_{4}+k_{2}^{2}\right)\left(54 m^{2} v^{2} k_{3} k_{4}-27 v^{2} k_{3} k_{4}-k_{2}^{2}\right)\left(27 m^{2} v^{2} k_{3} k_{4}+27 v^{2} k_{3} k_{4}+k_{2}^{2}\right)=0 .
$$

The exact soliton solution to the equation will thus be determined as follows

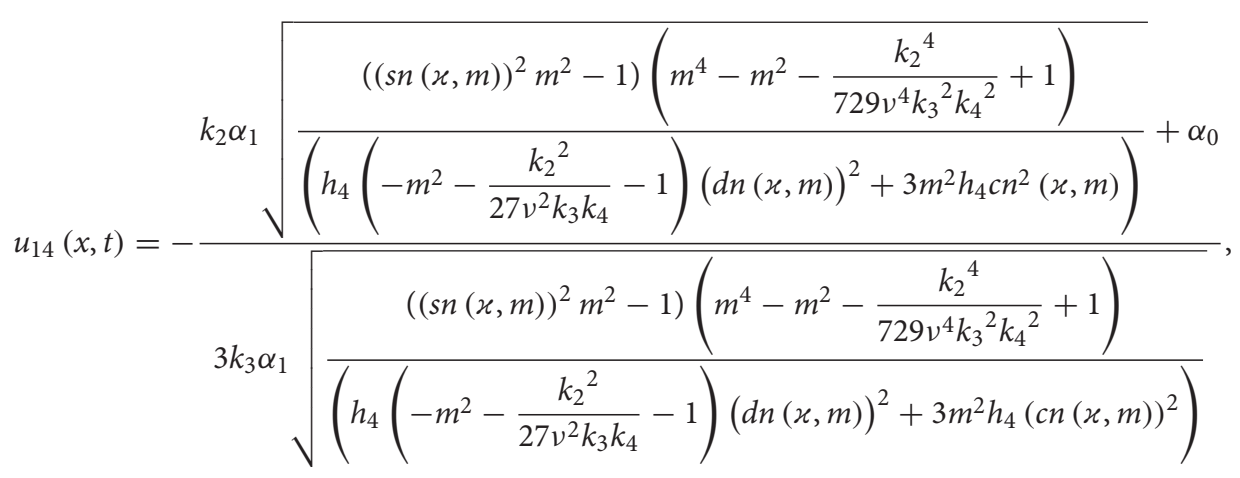

where

$$
\varkappa=\frac{-2 v k_{2}^{2}}{27 k_{3}} x-v t
$$

Using No. 7 in Table 1 we have

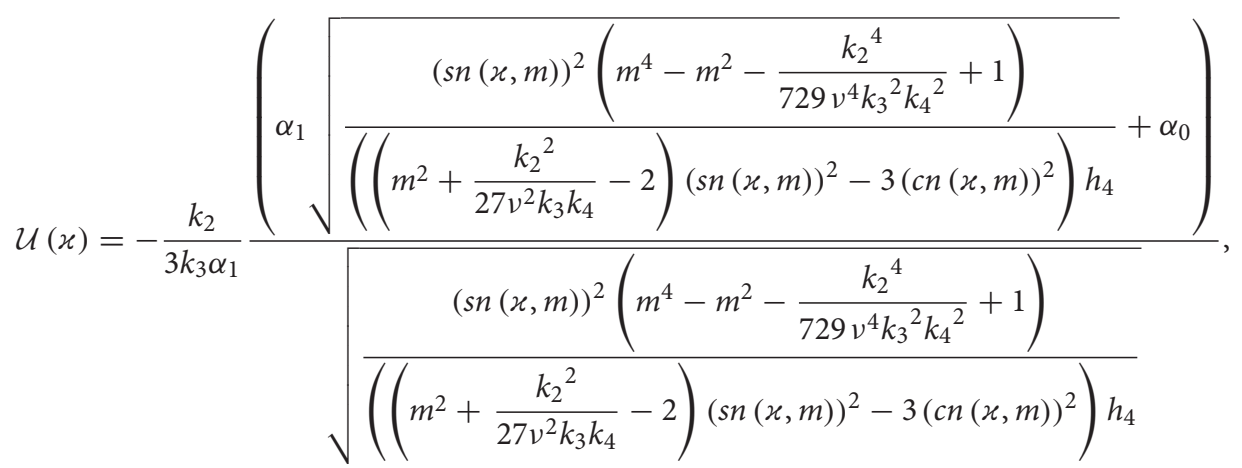

provided that

$$
\left(27 m^{2} v^{2} k_{3} k_{4}-54 v^{2} k_{3} k_{4}+k_{2}^{2}\right)\left(27 m^{2} v^{2} k_{3} k_{4}+27 v^{2} k_{3} k_{4}+k_{2}^{2}\right)\left(54 m^{2} v^{2} k_{3} k_{4}-27 v^{2} k_{3} k_{4}-k_{2}^{2}\right)=0 .
$$


The exact soliton solution to the equation will thus be determined as follows

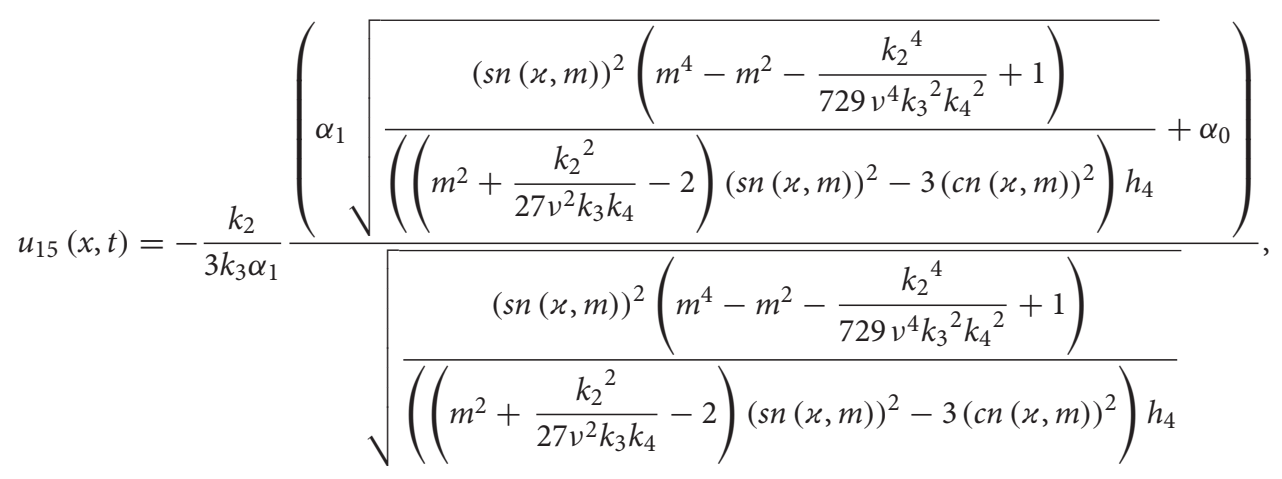

where

$$
x=\frac{-2 v k_{2}^{2}}{27 k_{3}} x-v t .
$$

Set 13: We attain

$$
\begin{gathered}
\mu=\frac{-2 v k_{2}^{2}}{27 k_{3}}, v=v, \alpha_{0}=\alpha_{0}, \alpha_{1}=-\frac{\beta_{1} k_{2}}{3 k_{3}}, \alpha_{2}=\alpha_{2}, \beta_{0}=0, \beta_{1}=\beta_{1}, \beta_{2}=0, h_{0}=-\frac{\alpha_{0}{ }^{2} k_{3}}{6 k_{4} \beta_{1}{ }^{2} v^{2}}, \\
h_{2}=-\frac{27 \alpha_{0} \alpha_{2} k_{3}{ }^{2}-\beta_{1}{ }^{2} k_{2}^{2}}{27 \beta_{1}{ }^{2} v^{2} k_{3} k_{4}}, h_{4}=-\frac{\alpha_{2}{ }^{2} k_{3}}{6 k_{4} \beta_{1}{ }^{2} v^{2}}, h_{6}=0 .
\end{gathered}
$$

Using No. 1 in Table 1 we have

$$
\mathcal{U}(x)=\frac{\left(\alpha_{0}-\frac{18(\operatorname{sn}(\xi, m))^{2} v^{2} k_{4}\left(m^{4}-m^{2}-\frac{\alpha_{0}{ }^{2} k_{2}^{2}}{81 \beta_{0}^{2} v^{4} k_{4}^{2}}+1\right)}{k_{2}\left(-3+\left(m^{2}-\frac{\alpha_{0} k_{2}}{9 \beta_{0} v^{2} k_{4}}+1\right)(s n(\xi, m))^{2}\right)}\right)}{\left(\sqrt{1+3 \beta_{1} \sqrt{6}}-\frac{(\operatorname{sn}(\xi, m))^{2} v^{2} k_{4} k_{3}\left(m^{4}-m^{2}-\frac{\alpha_{0}{ }^{2} k_{2}^{2}}{81 \beta_{0}^{2} v^{4} k_{4}^{2}}+1\right)}{\beta_{1}^{2} k_{2}^{2}\left(-3+\left(m^{2}-\frac{\alpha_{0} k_{2}}{9 \beta_{0} v^{2} k_{4}}+1\right)(s n(\xi, m))^{2}\right)}\right)},
$$

provided that

$$
\left(9 m^{2} \beta_{0} v^{2} k_{4}-18 \beta_{0} v^{2} k_{4}-\alpha_{0} k_{2}\right)\left(9 m^{2} \beta_{0} v^{2} k_{4}+9 \beta_{0} v^{2} k_{4}-\alpha_{0} k_{2}\right)\left(18 m^{2} \beta_{0} v^{2} k_{4}-9 \beta_{0} v^{2} k_{4}+\alpha_{0} k_{2}\right)=0
$$

The exact soliton solution to the equation will thus be determined as follows

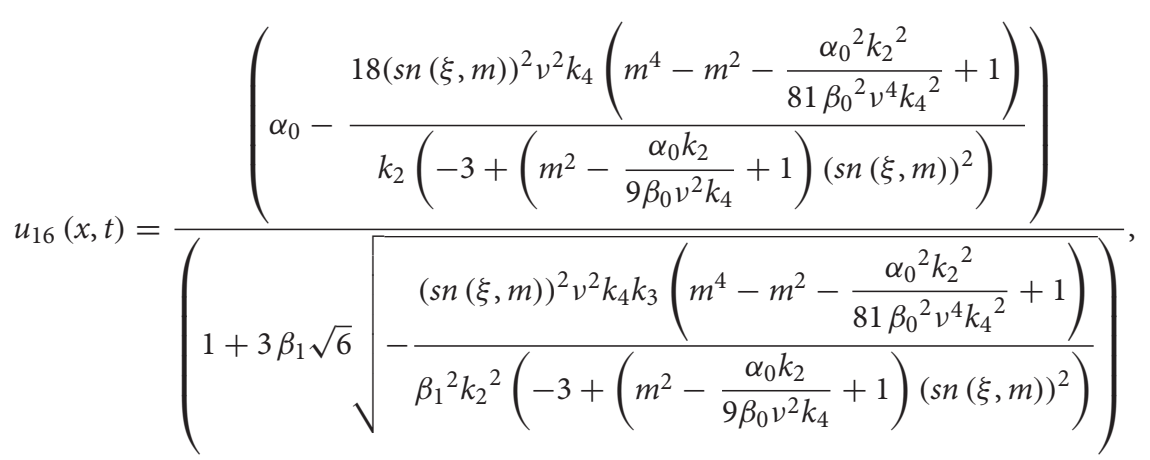


where

$$
\varkappa=\frac{-2 v k_{2}^{2}}{27 k_{3}} x-v t
$$

Using No. 8 in Table 1 we have

$$
\mathcal{U}(\varkappa)=-\frac{k_{2}}{3 k_{3} \alpha_{1}} \frac{\left(\alpha_{1} \frac{(\operatorname{sn}(\varkappa, m))^{2}}{h_{4}} \frac{\left(m^{4}-m^{2}-\frac{k_{2}{ }^{4}}{729 v^{4} k_{3}{ }^{2} k_{4}{ }^{2}}+1\right)}{\left.\sqrt{\left.\frac{(\operatorname{sn}(\varkappa, m))^{2}}{h_{4}}\left(m^{4}+\frac{k_{2}{ }^{2}}{27 v^{2} k_{3} k_{4}}+1\right)(\operatorname{sn}(\varkappa, m))^{2}\right)}+m^{2}-\frac{k_{2}{ }^{4}}{729 v^{4} k_{3}{ }^{2} k_{4}{ }^{2}}+1\right)\left(-3+\left(m^{2}+\frac{k_{2}{ }^{2}}{27 v^{2} k_{3} k_{4}}+1\right)(\operatorname{sn}(\varkappa, m))^{2}\right)}\right.}{\sqrt{\left.m^{2}\right)}}
$$

provided that

$$
\left(9 m^{2} \beta_{0} v^{2} k_{4}-18 \beta_{0} v^{2} k_{4}-\alpha_{0} k_{2}\right)\left(9 m^{2} \beta_{0} v^{2} k_{4}+9 \beta_{0} v^{2} k_{4}-\alpha_{0} k_{2}\right)\left(18 m^{2} \beta_{0} v^{2} k_{4}-9 \beta_{0} v^{2} k_{4}+\alpha_{0} k_{2}\right)=0 .
$$

The exact soliton solution to the equation will thus be determined as follows

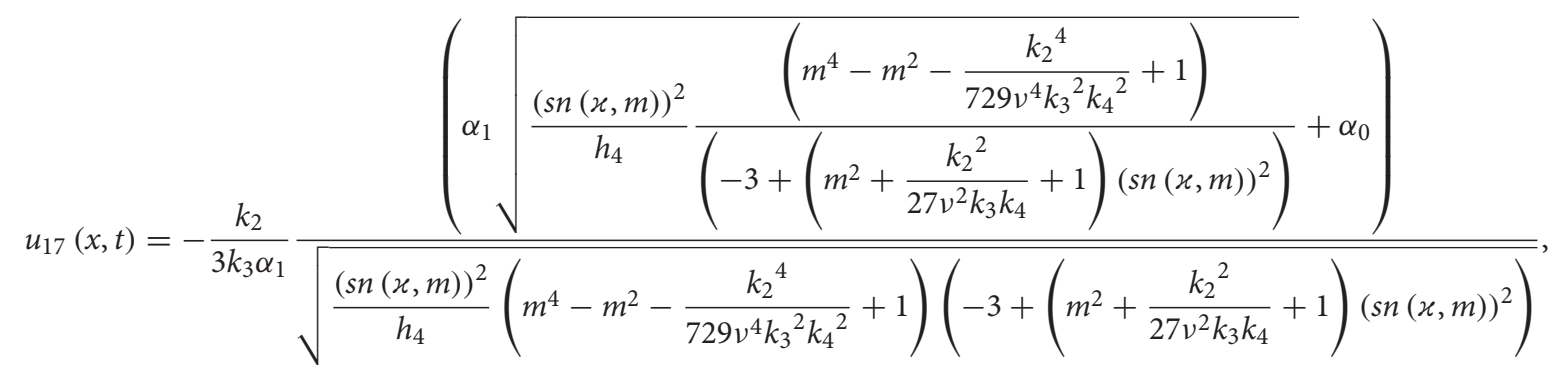

where

$$
\varkappa=\frac{v\left(27 k_{1} k_{3}-2 k_{2}^{2}\right) x}{27 k_{3}}-v t .
$$

Set 14: We attain

$$
\begin{gathered}
\mu=\frac{v\left(432 v^{4} \beta_{0}{ }^{4} h_{6}{ }^{2} k_{3} k_{4}{ }^{2}+4 v^{2} \beta_{0}{ }^{2} \beta_{2}{ }^{2} h_{6} k_{2}{ }^{2} k_{4}-4 v^{2} \beta_{0} \beta_{2}{ }^{3} h_{4} k_{2}{ }^{2} k_{4}+\beta_{2}{ }^{4} k_{1} k_{2}{ }^{2}\right)}{k_{2}{ }^{2} \beta_{2}{ }^{4}}, v=v, \\
\alpha_{0}=\frac{36 v^{2} \beta_{0}{ }^{3} h_{6} k_{4}}{\beta_{2}{ }^{2} k_{2}}, \alpha_{1}=0, \alpha_{2}=-\frac{36 v^{2} \beta_{0}{ }^{2} h_{6} k_{4}}{\beta_{2} k_{2}}, \beta_{0}=\beta_{0}, \beta_{1}=0, \beta_{2}=\beta_{2}, \\
h_{0}=\frac{\beta_{0}{ }^{2}\left(2 \beta_{0} h_{6}+\beta_{2} h_{4}\right)}{\beta_{2}{ }^{3}}, h_{2}=-\frac{\beta_{0}\left(216 v^{2} \beta_{0}{ }^{3} h_{6}{ }^{2} k_{3} k_{4}-\beta_{0} \beta_{2}{ }^{2} h_{6} k_{2}{ }^{2}-2 \beta_{2}{ }^{3} h_{4} k_{2}{ }^{2}\right)}{k_{2}{ }^{2} \beta_{2}{ }^{4}}, h_{4}=h_{4}, h_{6}=h_{6} .
\end{gathered}
$$

Using No. 1 in Table 1 we have

$$
\mathcal{U}(\varkappa)=-\frac{36 v^{2} h_{6} k_{4}\left(\left(\left(m^{4}-m^{2}-\Delta^{2}+1\right) \beta_{2}-h_{4}\left(m^{2}-\Delta+1\right)\right)(s n(\xi, m))^{2}+3 h_{4}\right)}{\beta_{2}{ }^{2} k_{2}\left(\left(\left(m^{4}-m^{2}-\Delta^{2}+1\right) \beta_{2}+h_{4}\left(m^{2}-\Delta+1\right)\right)(s n(\xi, m))^{2}-3 h_{4}\right)},
$$

where $\Delta=\frac{\beta_{0}\left(216 v^{2} \beta_{0}{ }^{3} h_{6}{ }^{2} k_{3} k_{4}-\beta_{0} \beta_{2}{ }^{2} h_{6} k_{2}{ }^{2}-2 \beta_{2}{ }^{3} h_{4} k_{2}{ }^{2}\right)}{k_{2}{ }^{2} \beta_{2}{ }^{4}}$, provided that one of following conditions holds 


$$
\begin{gathered}
\left(-216 v^{2} \beta_{0}{ }^{4} h_{6}{ }^{2} k_{3} k_{4}+m^{2} k_{2}{ }^{2} \beta_{2}{ }^{4}+\beta_{0}{ }^{2} \beta_{2}{ }^{2} h_{6} k_{2}{ }^{2}\right. \\
\left.+2 \beta_{0} \beta_{2}{ }^{3} h_{4} k_{2}{ }^{2}-2 k_{2}{ }^{2} \beta_{2}{ }^{4}\right)=0, \\
\left(-216 v^{2} \beta_{0}{ }^{4} h_{6}{ }^{2} k_{3} k_{4}+m^{2} k_{2}{ }^{2} \beta_{2}{ }^{4}\right. \\
\left.+\beta_{0}{ }^{2} \beta_{2}{ }^{2} h_{6} k_{2}{ }^{2}+2 \beta_{0} \beta_{2}{ }^{3} h_{4} k_{2}{ }^{2}+k_{2}{ }^{2} \beta_{2}{ }^{4}\right)=0, \\
\left(216 v^{2} \beta_{0}{ }^{4} h_{6}{ }^{2} k_{3} k_{4}+2 m^{2} k_{2}{ }^{2} \beta_{2}{ }^{4}-\beta_{0}{ }^{2} \beta_{2}{ }^{2} h_{6} k_{2}{ }^{2}\right. \\
\left.-2 \beta_{0} \beta_{2}{ }^{3} h_{4} k_{2}{ }^{2}-k_{2}{ }^{2} \beta_{2}{ }^{4}\right) h_{4}{ }^{2}=0 .
\end{gathered}
$$

The exact soliton solution to the equation will thus be determined as follows

$$
\begin{gathered}
36 v^{2} h_{6} k_{4}\left(\left(\left(m^{4}-m^{2}-\Delta^{2}+1\right) \beta_{2}\right.\right. \\
u_{18}(x, t)=-\frac{\left.\left.-h_{4}\left(m^{2}-\Delta+1\right)\right)(s n(\xi, m))^{2}+3 h_{4}\right)}{\beta_{2}{ }^{2} k_{2}\left(\left(\left(m^{4}-m^{2}-\Delta^{2}+1\right) \beta_{2}\right.\right.} \\
\left.\left.+h_{4}\left(m^{2}-\Delta+1\right)\right)(s n(\xi, m))^{2}-3 h_{4}\right)
\end{gathered}
$$

where

$$
\varkappa=\frac{v\left(432 v^{4} \beta_{0}{ }^{4} h_{6}{ }^{2} k_{3} k_{4}{ }^{2}+4 v^{2} \beta_{0}{ }^{2} \beta_{2}{ }^{2} h_{6} k_{2}{ }^{2} k_{4}\right.}{\left.-4 v^{2} \beta_{0} \beta_{2}{ }^{3} h_{4} k_{2}{ }^{2} k_{4}+\beta_{2}{ }^{4} k_{1} k_{2}{ }^{2}\right) x}-v t .
$$

Using No. 5 in Table 1 we have

$$
\begin{aligned}
& \mathcal{U}(\varkappa)= \\
& -\frac{36 v^{2} h_{6} k_{4}\left(-3(c n(\xi, m))^{2} m^{2} h_{4}\right.}{\beta_{2}{ }^{2} k_{2}\left(3(c n(\xi, m))^{2} m^{2} h_{4}+\left(m^{4}-m^{2}-\Delta^{2}+1\right) \beta_{2}\right.}, \\
& \left.\quad+\left(-2 m^{2}-\Delta+1\right) h_{4}\right)
\end{aligned}
$$

where $\Delta=\frac{\beta_{0}\left(216 v^{2} \beta_{0}{ }^{3} h_{6}{ }^{2} k_{3} k_{4}-\beta_{0} \beta_{2}{ }^{2} h_{6} k_{2}{ }^{2}-2 \beta_{2}{ }^{3} h_{4} k_{2}{ }^{2}\right)}{k_{2}{ }^{2} \beta_{2}{ }^{4}}$, provided that one of following conditions holds

$$
\begin{gathered}
\left(-216 v^{2} \beta_{0}{ }^{4} h_{6}{ }^{2} k_{3} k_{4}+m^{2} k_{2}{ }^{2} \beta_{2}{ }^{4}+\beta_{0}{ }^{2} \beta_{2}{ }^{2} h_{6} k_{2}{ }^{2}\right. \\
\left.+2 \beta_{0} \beta_{2}{ }^{3} h_{4} k_{2}{ }^{2}-2 k_{2}{ }^{2} \beta_{2}{ }^{4}\right)=0, \\
\left(-216 v^{2} \beta_{0}{ }^{4} h_{6}{ }^{2} k_{3} k_{4}+m^{2} k_{2}{ }^{2} \beta_{2}{ }^{4}+\beta_{0}{ }^{2} \beta_{2}{ }^{2} h_{6} k_{2}{ }^{2}\right. \\
\left.+2 \beta_{0} \beta_{2}{ }^{3} h_{4} k_{2}{ }^{2}+k_{2}{ }^{2} \beta_{2}{ }^{4}\right)=0, \\
\left(216 v^{2} \beta_{0}{ }^{4} h_{6}{ }^{2} k_{3} k_{4}+2 m^{2} k_{2}{ }^{2} \beta_{2}{ }^{4}-\beta_{0}{ }^{2} \beta_{2}{ }^{2} h_{6} k_{2}{ }^{2}\right. \\
\left.-2 \beta_{0} \beta_{2}{ }^{3} h_{4} k_{2}{ }^{2}-k_{2}{ }^{2} \beta_{2}{ }^{4}\right) h_{4}{ }^{2}=0 .
\end{gathered}
$$

The exact soliton solution to the equation will thus be determined as follows

$u_{18}(x, t)$

$$
\begin{gathered}
36 v^{2} h_{6} k_{4}\left(-3(c n(\xi, m))^{2} m^{2} h_{4}\right. \\
=-\frac{\left.+\left(m^{4}-m^{2}-\Delta^{2}+1\right) \beta_{2}+h_{4}\left(2 m^{2}+\Delta-1\right)\right)}{\beta_{2}{ }^{2} k_{2}\left(3(c n(\xi, m))^{2} m^{2} h_{4}+\left(m^{4}-m^{2}-\Delta^{2}+1\right) \beta_{2}\right.}, \\
\left.+\left(-2 m^{2}-\Delta+1\right) h_{4}\right)
\end{gathered}
$$

where

$$
\varkappa=\frac{v\left(432 v^{4} \beta_{0}{ }^{4} h_{6}{ }^{2} k_{3} k_{4}{ }^{2}+4 v^{2} \beta_{0}{ }^{2} \beta_{2}{ }^{2} h_{6} k_{2}{ }^{2} k_{4}\right.}{\left.-4 v^{2} \beta_{0} \beta_{2}{ }^{3} h_{4} k_{2}{ }^{2} k_{4}+\beta_{2}{ }^{4} k_{1} k_{2}{ }^{2}\right) x}-v t .
$$

Likewise, other new families of solutions are obtained by following steps similar to the above using the following sets of parameters.

Set 15: We attain

$$
\begin{aligned}
& \mu=\frac{-2 \alpha_{2}{ }^{2} k_{2}{ }^{2} v}{162 v^{2} \beta_{1}^{2} h_{4} k_{4}+27 \alpha_{2}{ }^{2} k_{3}}, v=v, \alpha_{0}=0, \\
& \alpha_{1}=0, \alpha_{2}=\alpha_{2}, \beta_{0}=0, \beta_{1}=\beta_{1},
\end{aligned}
$$

$$
\begin{aligned}
& \beta_{2}=-\frac{18 v^{2} \beta_{1}{ }^{2} h_{4} k_{4}+3 \alpha_{2}{ }^{2} k_{3}}{2 \alpha_{2} k_{2}}, h_{0}=0, \\
& h_{2}=-\frac{2 \alpha_{2}{ }^{2} k_{2}{ }^{2}}{27 k_{4}\left(6 v^{2} \beta_{1}{ }^{2} h_{4} k_{4}+\alpha_{2}{ }^{2} k_{3}\right) v^{2}}, h_{4}=h_{4}, h_{6}=0 .
\end{aligned}
$$

\section{Set 16: We attain}

$$
\begin{gathered}
\mu=\frac{v\left(-3456 v^{4} \beta_{0}{ }^{4} h_{4}{ }^{2} k_{2}{ }^{2} k_{3} k_{4}{ }^{2}-48 v^{2} \beta_{0}{ }^{2} \beta_{1}{ }^{2} h_{4} k_{2}{ }^{4} k_{4}\right)}{\left(216 v^{2} \beta_{0}{ }^{2} h_{4} k_{4} k_{3}+\beta_{1}{ }^{2} k_{2}{ }^{2}\right)^{2}}, \\
v=v, \alpha_{0}=-\frac{576 \beta_{1}{ }^{2} v^{2} \beta_{0}{ }^{3} h_{4} k_{4} k_{2}{ }^{3}}{\left(216 v^{2} \beta_{0}{ }^{2} h_{4} k_{4} k_{3}+\beta_{1}{ }^{2} k_{2}{ }^{2}\right)^{2}}, \\
\alpha_{1}=-\frac{144 v^{2} \beta_{0}{ }^{2} \beta_{1} h_{4} k_{4} k_{2}}{216 v^{2} \beta_{0}{ }^{2} h_{4} k_{4} k_{3}+\beta_{1}{ }^{2} k_{2}{ }^{2}}, \alpha_{2}=0, \beta_{0}=\beta_{0}, \beta_{1}=\beta_{1}, \\
\beta_{2}=\frac{216 v^{2} \beta_{0}{ }^{2} h_{4} k_{4} k_{3}+\beta_{1}{ }^{2} k_{2}{ }^{2}}{4 k_{2}{ }^{2} \beta_{0}}, \\
h_{0}=\frac{144 \beta_{0}{ }^{4} h_{4}\left(5184 v^{4} \beta_{0}{ }^{4} h_{4}{ }^{2} k_{3}{ }^{2} k_{4}{ }^{2}-144 v^{2} \beta_{0}{ }^{2} \beta_{1}{ }^{2} h_{4} k_{2}{ }^{2} k_{3} k_{4}\right.}{\left.+\beta_{1}{ }^{4} k_{2}{ }^{4}\right) k_{2}{ }^{4}} \\
h_{2}=\frac{24 h_{4}\left(72 v^{2} \beta_{0}{ }^{2} h_{4} k_{4} k_{3}-\beta_{1}{ }^{2} k_{2}{ }^{2}\right) k_{2}{ }^{2} \beta_{0}{ }^{2}}{\left(216 v^{2} \beta_{0}{ }^{2} h_{4} k_{4} k_{0}{ }^{2} h_{4} k_{3} k_{4} k_{3}+\beta_{1}{ }^{2} k_{2}{ }^{2}\right)^{2}} .
\end{gathered}
$$


Set 17: We attain

$$
\begin{aligned}
& \mu=\frac{-2 v k_{2}^{2}}{27 k_{3}}, v=v, \alpha_{0}=-\frac{\beta_{0} k_{2}}{3 k_{3}}, \alpha_{1}=\alpha_{1}, \\
& \alpha_{2}=\frac{3\left(96 v^{2} \beta_{0}^{2} h_{4} k_{4}-\alpha_{1}^{2} k_{3}\right)}{8 \beta_{0} k_{2}}, \beta_{0}=\beta_{0}, \beta_{1}=0, \\
& \beta_{2}=-\frac{9 k_{3}\left(96 v^{2} \beta_{0}^{2} h_{4} k_{4}-\alpha_{1}^{2} k_{3}\right)}{8 k_{2}^{2} \beta_{0}},
\end{aligned}
$$

$$
\begin{aligned}
& h_{0}=\frac{64 k_{2}{ }^{4} h_{4} \beta_{0}{ }^{4}}{81 k_{3}^{2}\left(96 v^{2} \beta_{0}^{2} h_{4} k_{4}-\alpha_{1}{ }^{2} k_{3}\right)^{2}}, \\
& h_{2}=-\frac{k_{2}^{2}\left(48 v^{2} \beta_{0}^{2} h_{4} k_{4}+\alpha_{1}^{2} k_{3}\right)}{27 k_{3} k_{4}\left(96 v^{2} \beta_{0}^{2} h_{4} k_{4}-\alpha_{1}{ }^{2} k_{3}\right) v^{2}}, h_{4}=h_{4}, h_{6}=0 .
\end{aligned}
$$

As can be seen, many varied sets of soliton solutions to Gardner's equation will be obtained by applying this method. In the structure of these solutions, rational, hyperbolic, trigonometric, exponential and Jacobi elliptical functions are used. The
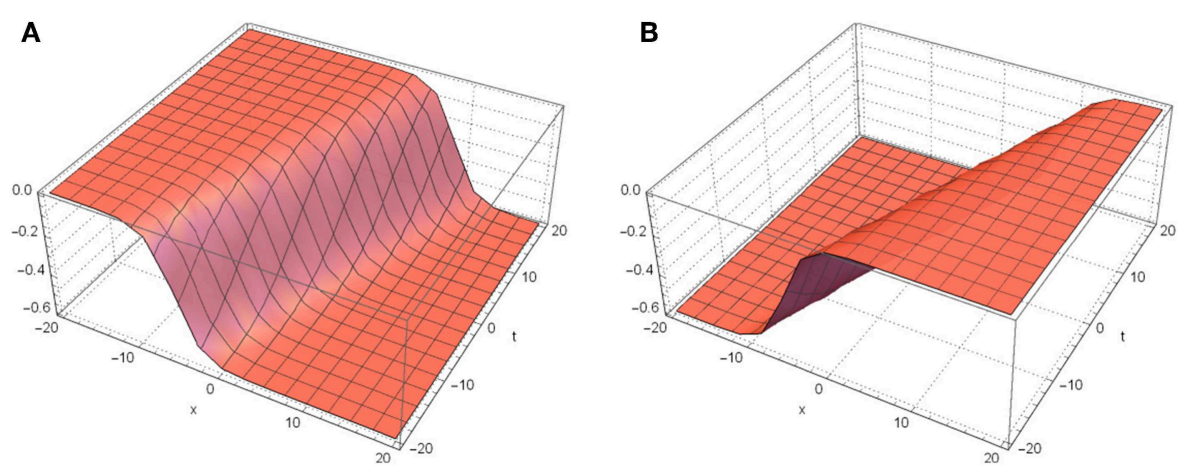

FIGURE 1 | Dynamic behavior of $u_{1}(x, t), u_{2}(x, t)$ for $k_{2}=1.2, k_{3}=2, k_{4}=-2$. (A) $u_{1}(x, t)$. (B) $u_{2}(x, t)$.
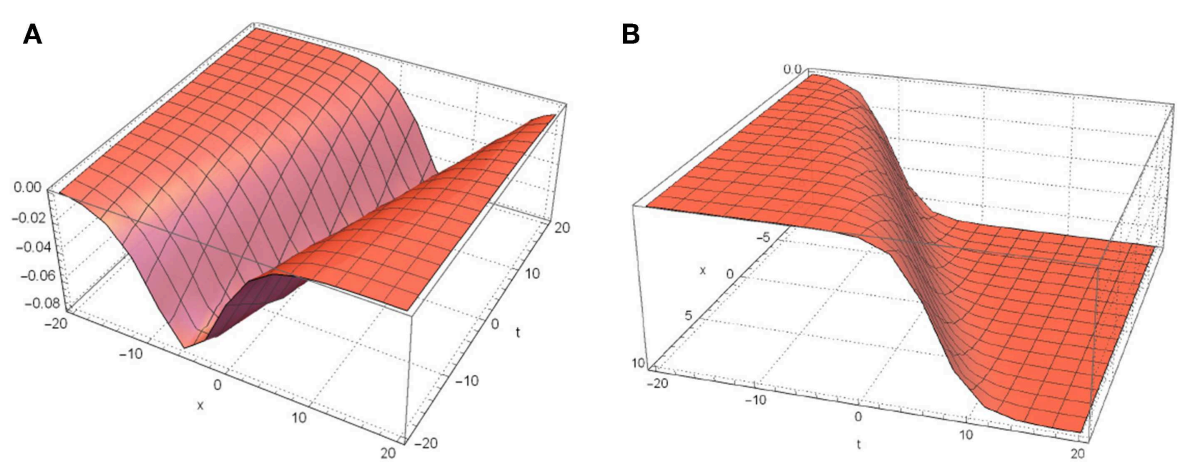

FIGURE 2 | Dynamic behavior of $u_{5}(x, t), u_{8}(x, t)$ for $k_{2}=1.2, k_{3}=2, k_{4}=-2$. (A) $u_{5}(x, t)$. (B) $u_{8}(x, t)$.
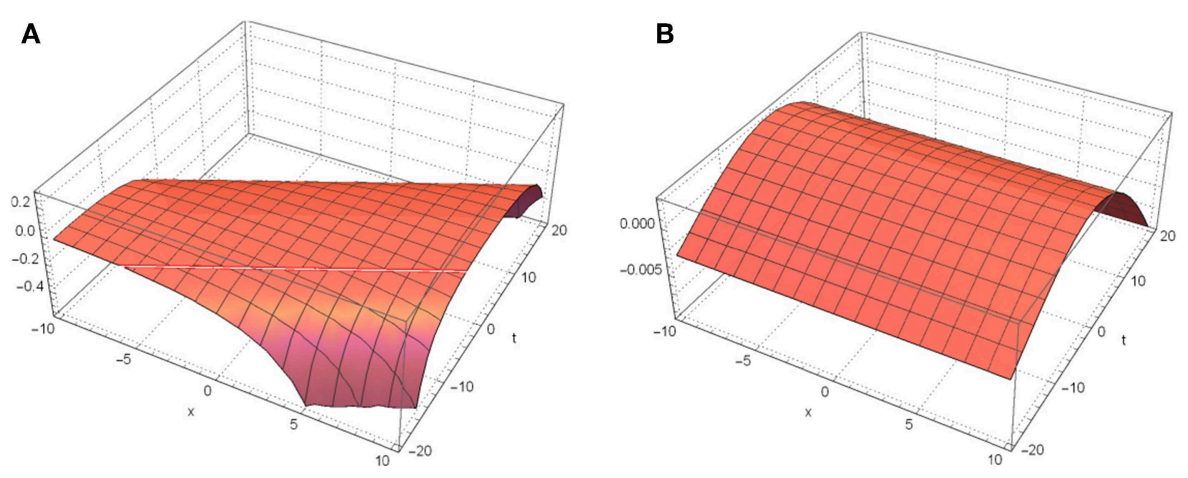

FIGURE 3 | Dynamic behavior of $u_{9}(x, t)$, and $u_{10}(x, t)$ for $k_{2}=1.1, k_{3}=-1.2, k_{4}=0.5$. (A) $u_{9}(x, t)$. (B) $u_{10}(x, t)$. 


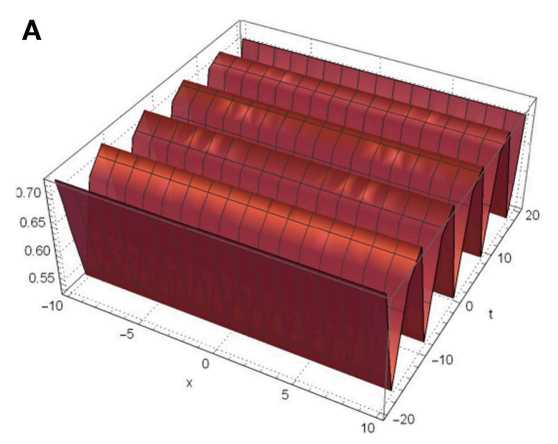

B

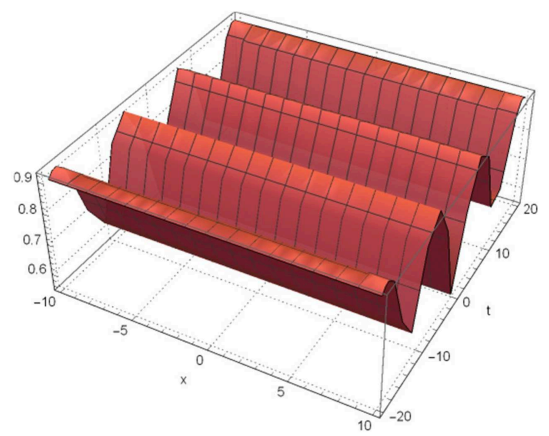

FIGURE 4 | Dynamic behavior of $\left|u_{11}(x, t)\right|$ for $k_{2}=0.8, k_{3}=-0.5, k_{4}=0.5$., and $\alpha_{1}=0.1$. (A) $m=0.5$ and $v=-\frac{2 k_{2}}{3 \sqrt{-6 k_{3} k_{4}}}$. (B) $m=0.8$ and $v=\frac{5 \sqrt{102} k_{2}}{306 \sqrt{k_{3} k_{4}}}$.
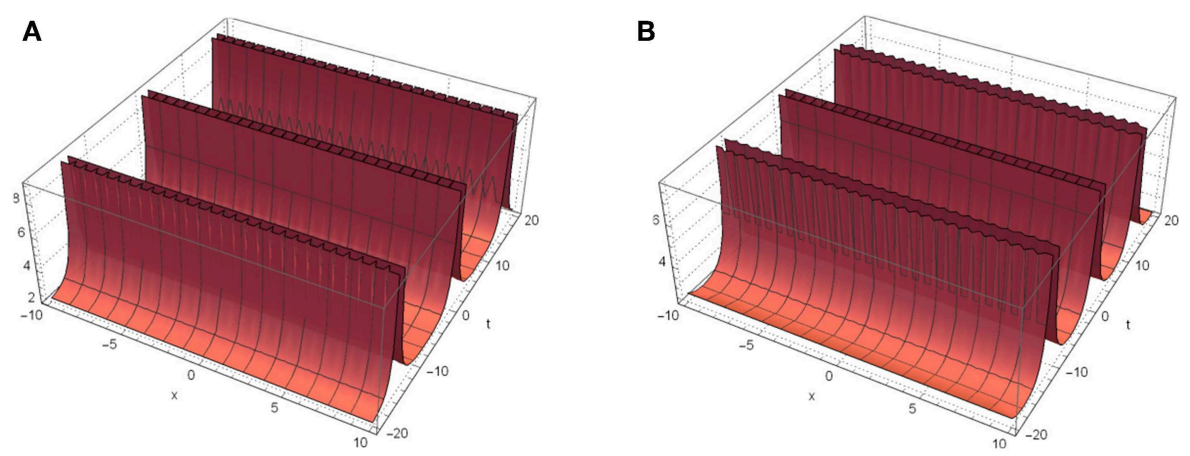

FIGURE 5 | Dynamic behavior of $\left|u_{12}(x, t)\right|$ for $k_{2}=0.3, k_{3}=-0.1, k_{4}=0.2$. (A) $m=0.2$ and $v=-\frac{5 \sqrt{3} k_{2}}{63 \sqrt{k_{3} k_{4}}}$. (B) $m=0.5$ and $v=-\frac{2 \sqrt{21} k_{2}}{63 \sqrt{k_{3} k_{4}}}$.
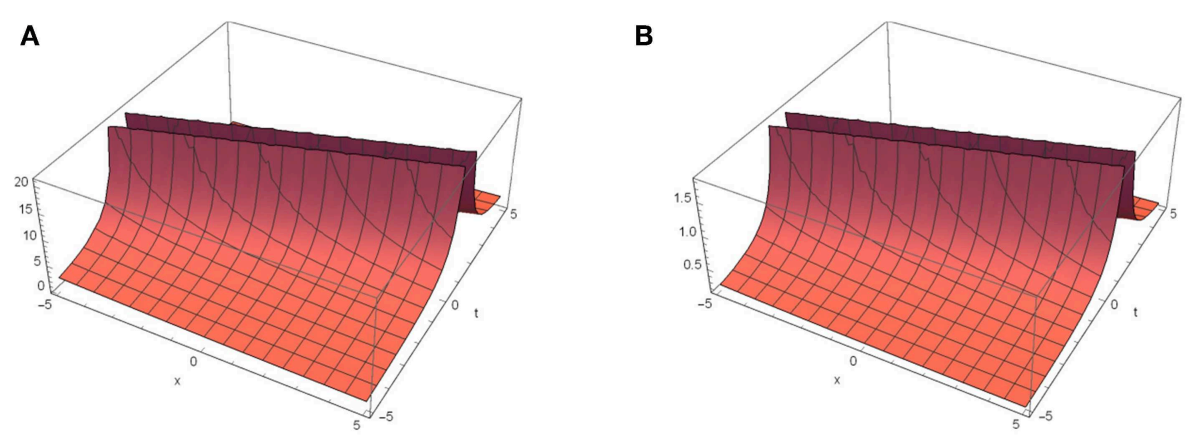

FIGURE 6 | Dynamic behavior of $\left|u_{15}(x, t)\right|$ for $k_{2}=0.2, k_{3}=-0.9, k_{4}=1$. (A) $m=0.3$ and $v=-\frac{10 k_{2}}{3 \sqrt{-246 k_{3} k_{4}}}$.

(B) $m=0.7$ and $v=-\frac{10 k_{2}}{3 \sqrt{-6 k_{3} k_{4}}}$.

correctness of all the obtained answers has been carefully examined. All of these soliton solutions are new findings presented for the first time in this article.

\section{GRAPHICAL REPRESENTATION}

We aimed to find new solutions for a given problem in Equation (1), and these new solutions should be described graphically. Thus, we present a graphical representation of some obtained solutions with the help of Mathematica in Figures 1-9. From these plots, some interesting and important physics phenomena can be observed.

\section{CONCLUSION}

In this manuscript, we have studied the Gardner equation with the help of two exact solution finder methods. A set of new exact solutions, including bright, kink, multi-soliton 
A

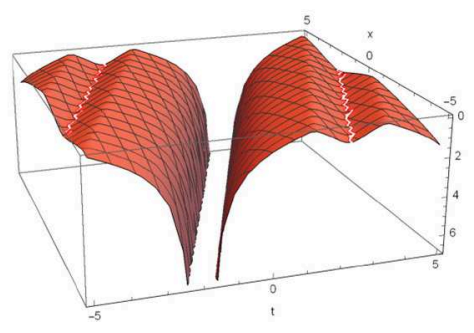

B



FIGURE $7 \mid$ Dynamic behavior of $\left|u_{16}(x, t)\right|$ for $k_{2}=0.1, k_{3}=0.7, k_{4}=-1.3$, and $\alpha_{2}=0.1, \beta_{1}=1$. (A) $m=0.1$ and $v=\frac{5 \sqrt{6} \sqrt{k_{3} k_{4}\left(27 \alpha_{0} \alpha_{2} k_{3}{ }^{2}-\beta_{1}{ }^{2} k_{2}{ }^{2}\right)}}{63 k_{3} k_{4} \beta_{1}}$. (B) $m=0.9$ and $\nu=\frac{10 \sqrt{-357 k_{3} k_{4}\left(27 \alpha_{0} \alpha_{2} k_{3}{ }^{2}-\beta_{1}{ }^{2} k_{2}{ }^{2}\right)}}{1071 k_{3} k_{4} \beta_{1}}$.

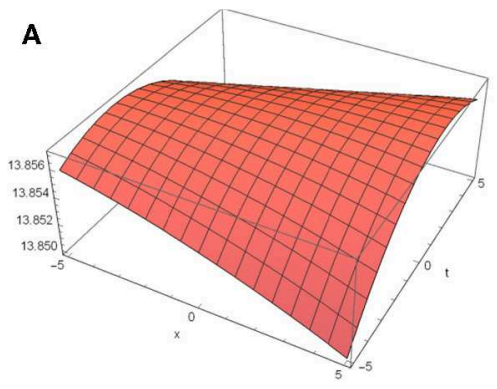

B

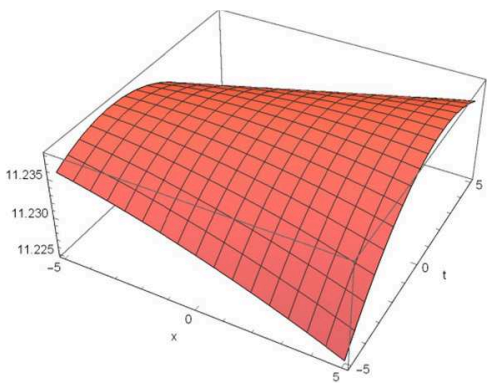

FIGURE 8 | Dynamic behavior of $\left|u_{18}(x, t)\right|$ for $k_{2}=0.1, k_{3}=0.7, k_{4}=-1.3$, and $\beta_{2}=0.1, h_{6}=1$. (A) $m=0.3$ and $v=\frac{\sqrt{3} \sqrt{k_{3} k_{4}\left(50 \beta_{0}{ }^{2} h_{6}+100 \beta_{0} \beta_{2} h_{4}+41 \beta_{2}{ }^{2}\right)} k_{2} \beta_{2}}{180 \beta_{0} h_{6} k_{3} k_{4}}$. (B) $m=0.8$ and $v=\frac{\sqrt{6} \sqrt{k_{3} k_{4}\left(25 \beta_{0}^{2} h_{6}+50 \beta_{0} \beta_{2} h_{4}-7 \beta_{2}^{2}\right)} k_{2} \beta_{2}}{180 \beta_{0}^{2} h_{0} k_{0} k_{4}}$.

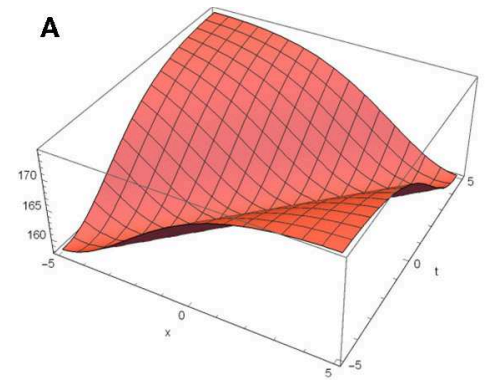

B



FIGURE 9 | Dynamic behavior of $\left|u_{19}(x, t)\right|$ for $k_{2}=0.7, k_{3}=0.4, k_{4}=-0.5$, and $\beta_{2}=h_{6}=1$. (A) $m=0.1$ and $v=\frac{\sqrt{3} \sqrt{k_{3} k_{4}\left(50 \beta_{0}{ }^{2} h_{6}+100 \beta_{0} \beta_{2} h_{4}+49 \beta_{2}{ }^{2}\right)} k_{2} \beta_{2}}{180 k_{3} k_{4} h_{6} \beta_{0}{ }^{2}}$. (B) $m=0.7$ and $v=\frac{\sqrt{3} \sqrt{k_{3} k_{4}\left(50 \beta_{0}{ }^{2} h_{6}+100 \beta_{0} \beta_{2} h_{4}+\beta_{2}{ }^{2}\right)} k_{2} \beta_{2}}{180 k_{3} k_{4} h_{6} \beta_{0}{ }^{2}}$.

solutions, and singular solitons were found corresponding to four parameters, namely $k_{1}, k_{2}, k_{3}$, and $k_{4}$. The dynamic behavior of the acquired solutions was also demonstrated to deeply understand the features of the non-linear model. In order to better their properties, we have drawn some 3-D graphs. To the best of the authors knowledge, all the acquired results are novel findings, and cannot be found in the previous works. This result verifies the power of two suggested methods. The main advantages of the method are that they are very simple and quite efficient for the estimation of the optical solutions of PDES. Moreover, the proposed approaches represent efficient methodologies to investigate the exact solutions of the non-linear PDEs.

\section{DATA AVAILABILITY STATEMENT}

The datasets generated for this study are available on request to the corresponding author. 


\section{AUTHOR CONTRIBUTIONS}

All authors listed have made a substantial, direct and intellectual contribution to the work, and approved it for publication.

\section{REFERENCES}

1. Biazar J, Ayati Z. Extension of the Exp-function method for systems of two-dimensional Burgers equations. Comput Math Appl. (2009) 58:2103-6. doi: 10.1016/j.camwa.2009.03.003

2. Zhaqilao, Qiao Z. Darboux transformation and explicit solutions for two integrable equations. J Math Anal Appl. (2011) 380:794-806. doi: 10.1016/j.jmaa.2011.01.078

3. Yang S, Hua C. Lie symmetry reductions and exact solutions of a coupled KdV-Burgers equation. Appl Math Comput. (2014) 234:579-83. doi: 10.1016/j.amc.2014.01.044

4. Younis M. A new approach for the exact solutions of nonlinear equations of fractional order via modified simple equation method. Appl Math. (2014) 5:1927-32. doi: 10.4236/am.2014.513186

5. Wang M. Exact solutions for a compound KdV-Burgers equation. Phys Lett A. (1996) 213:279-87. doi: 10.1016/0375-9601(96)00103-x

6. Djilali S. Impact of prey herd shape on the predator-prey interaction. Chaos Solit Fract. (2019) 120:139-48. doi: 10.1016/j.chaos.2019.01.022

7. Djilali S, Bentout S. Spatiotemporal patterns in a diffusive predatorprey model with prey social behavior. Acta Appl Math. (2019). doi: 10.1007/s10440-019-00291-z. [Epub ahead of print].

8. Djilali S. Herd behavior in a predator-prey model with spatial diffusion: bifurcation analysis and Turing instability. J Appl Math Comput. (2017) 58:125-49. doi: 10.1007/s12190-017-1137-9

9. Djilali S, Touaoula TM, Miri SEH. A heroin epidemic model: very general non linear incidence, treat-age, and global stability. Acta Appl Math. (2017) 152:171-94. doi: 10.1007/s10440-017-0117-2

10. Goufo EFD, Kumar S, Mugisha SB. Similarities in a fifth-order evolution equation with and with no singular kernel. Chaos Solit Fract. (2020) 130:109467. doi: 10.1016/j.chaos.2019.109467

11. Odibat Z, Kumar S. A robust computational algorithm of homotopy asymptotic method for solving systems of fractional differential equations. $J$ Comput Nonlin Dyn. (2019) 14:081004. doi: 10.1115/1.4043617

12. El-Ajou A, Oqielat MN, Al-Zhour Z, Kumar S, Momani S. Solitary solutions for time-fractional nonlinear dispersive PDEs in the sense of conformable fractional derivative. Chaos. (2019) 29:093102. doi: 10.1063/1.5100234

13. Cattani C, Rushchitskii YY. Cubically nonlinear elastic waves: wave equations and methods of analysis. Int Appl Mech. (2003) 39:1115-45. doi: 10.1023/b:inam.0000010366.48158.48

14. Yang AM, Zhang YZ, Cattani C, Xie GN, Rashidi MM, Zhou YJ, et al. Application of local fractional series expansion method to solve KleinGordon equations on cantor sets. Abstr Appl Anal. (2014) 2014:1-6. doi: $10.1155 / 2014 / 372741$

15. Cattani C. Haar wavelet-based technique for sharp jumps classification. Math Comput Model. (2004) 39:255-78. doi: 10.1016/s0895-7177(04)90010-6

16. Cattani C. Harmonic wavelet solutions of the Schrodinger equation. Int J Fluid Mech Res. (2003) 30:463-72. doi: 10.1615/interjfluidmechres.v30.i5.10

17. Cattani C, Sulaiman TA, Baskonus HM, Bulut H. On the soliton solutions to the Nizhnik-Novikov-Veselov and the Drinfel'd-Sokolov systems. Opt Quant Electr. (2018) 50:138. doi: 10.1007/s11082-018-1406-3

18. Avazzadeh Z, Heydari MH, Cattani C. Legendre wavelets for fractional partial integro-differential viscoelastic equations with weakly singular kernels. Eur Phys J Plus. (2019) 134:368. doi: 10.1140/epjp/i2019-12743-6

\section{FUNDING}

This work was supported by JMDC (100\%.2019.FPHY.827).
19. Griffiths GW, Shiesser WE. Traveling Wave Analysis of Partial Differential Equations. Cambridge, MA: Elsevier (2012). doi: 10.1016/c2009-0-64536-0

20. Wazwaz AM. Soliton solutions for two three plus one dimensional nonintegrable KdV-type equations. Math Comput Model. (2012) 55:1845-8. doi: $10.1016 /$ j.mcm.2011.11.082

21. Wazwaz AM. Solitons and singular solitons for the Gardner-KP equation. Appl Math Comput. (2008) 204:162-9. doi: 10.1016/j.amc.200 8.06.011

22. Krishnan EV, Triki H, Labidi M, Biswas A. A study of shallow water waves with Gardner's equation. Nonlin Dyn. (2011) 66:497-507. doi: 10.1007/s11071-010-9928-7

23. Kumar M, Tanwar DV. On Lie symmetries and invariant solutions of (2+1)-dimensional Gardner equation. Commun Nonlin Sci Numer Simul. (2019) 69:45-57. doi: 10.1016/j.cnsns.2018.09.009

24. Fei J, Cao W, Ma Z. Nonlocal symmetries and explicit solutions for the Gardner equation. Appl Math Comput. (2017) 314:293-8. doi: 10.1016/j.amc.2017.07.002

25. kai Liu Y, Li B. Nonlocal symmetry and exact solutions of the (2+1)-dimensional Gardner equation. Chin J Phys. (2016) 54:718-23. doi: 10.1016/j.cjph.2016.05.014

26. Betchewe G, Victor KK, Thomas BB, Crepin KT. New solutions of the Gardner equation: analytical and numerical analysis of its dynamical understanding. Appl Math Comput. (2013) 223:377-88. doi: 10.1016/j.amc.2013.08.028

27. Alejo MA. On the ill-posedness of the Gardner equation. J Math Anal Appl. (2012) 396:256-60. doi: 10.1016/j.jmaa.2012.06.018

28. Cao D. The classification of the single traveling wave solutions to the time-fraction Gardner equation. Chin J Phys. (2019) 59:379-92. doi: 10.1016/j.cjph.2019.03.003

29. Ghanbari B, Inc M. A new generalized exponential rational function method to find exact special solutions for the resonance nonlinear Schrödinger equation. Eur Phys J Plus. (2018) 133:142. doi: 10.1140/epjp/i201811984-1

30. Osman MS, Ghanbari B, Machado JAT. New complex waves in nonlinear optics based on the complex Ginzburg-Landau equation with Kerr law nonlinearity. Eur Phys J Plus. (2019) 134:20. doi: 10.1140/epjp/i2019-1 2442-4

31. Ghanbari B, Baleanu D, Qurashi MA. New exact solutions of the generalized Benjamin-Bona-Mahony equation. Symmetry. (2018) 11:20. doi: 10.3390/sym 11010020

32. Ghanbari B, Baleanu D. A novel technique to construct exact solutions for nonlinear partial differential equations. Eur Phys J Plus. (2019) 134:506 doi: 10.1140/epjp/i2019-13037-9

Conflict of Interest: The authors declare that the research was conducted in the absence of any commercial or financial relationships that could be construed as a potential conflict of interest.

Copyright (c) 2019 Ghanbari and Baleanu. This is an open-access article distributed under the terms of the Creative Commons Attribution License (CC BY). The use, distribution or reproduction in other forums is permitted, provided the original author(s) and the copyright owner(s) are credited and that the original publication in this journal is cited, in accordance with accepted academic practice. No use, distribution or reproduction is permitted which does not comply with these terms. 